\title{
DEVISING A VISUAL INSPECTION SYSTEM FOR CANAL TUNNELS: PRELIMINARY STUDIES
}

\author{
J.-L. Albert ${ }^{a}$, P. Charbonnier ${ }^{a}$, P. Chavant ${ }^{a, b}$, P. Foucher $^{a}$, V. Muzet ${ }^{a}$, D. Prybyla ${ }^{a}$, T. Perrin $^{a}$, \\ P. Grussenmeyer ${ }^{b}$, S. Guillemin ${ }^{b}$, M. Koehl ${ }^{b}$ \\ ${ }^{a}$ CETE de l'Est, LRPC de Strasbourg, 11 rue Jean Mentelin, 67035 Strasbourg, France \\ \{Jean-Louis.Albert,Pierre.Charbonnier,Philippe.Foucher,Valerie.Muzet, Davy.Prybyla,Thibaut.Perrin\}@ developpement-durable.gouv.fr \\ ${ }^{b}$ ICube Laboratory UMR 7357, Photogrammetry and Geomatics Group, \\ INSA de Strasbourg, 24 Bd de la Victoire, 67084 Strasbourg, France \\ \{pierre.grussenmeyer,samuel.guillemin,mathieu.koehl\}@insa-strasbourg.fr
}

KEY WORDS: Photogrammetry, Cultural Heritage, Recording, Surveying, Inspection, Imagery, Multisensor, Positioning

\begin{abstract}
:
In France, most tunnel canals were built during the 19th and 20th centuries. Maintaining them is not only a matter of heritage preservation but also a question of security. Inspecting tunnel canals is difficult and time consuming, which motivates the development of an image-based surveying system, as already exists for railway or road tunnels. However, while the imaging configuration is similar, referencing the data acquisition device is more difficult in the case of underground waterways, due to the drifts of the inspection barge. In this paper, we introduce the recording prototype we have designed and report the results of the test that were performed in an underground waterway, Niderviller's tunnel, to assess the feasibility of the system. In particular, we give details on the imaging system design. We also analyze the pros and cons of each location method, in terms of costs, practicability, computational burden and accuracy.
\end{abstract}

\section{INTRODUCTION}

There are 31 canal tunnels in service in France, totalling $42 \mathrm{~km}$ of underground waterway, among which the longest measures 5670 $\mathrm{m}$. They were mostly built during the 19 th and 20 th centuries. Their size is variable; however most of them are suited to the socalled Freycinet gauge (maximum $38.5 \mathrm{~m}$ long and $5.05 \mathrm{~m}$ wide boats). Many types of transports take canal tunnels: goods (including hazardous materials), passengers (tourism and pleasure boats), and other users (e.g. cyclists or pedestrian on ledges). Hence, keeping tunnels in good condition is not only a matter of heritage preservation, but also a security issue.

In this context, Voies Navigables de France (the French operator of waterways), the Centre d'Etudes des Tunnels (CETU) and the CETE de l'Est, in collaboration with the Photogrammetry and Geomatics Group (INSA) have begun a study for the development of a visual inspection system dedicated to canal tunnels. This project aims at devising a prototype for acquiring images of the tunnel vaults and side walls, from a barge. These image sequences should be browsed and analyzed in terms of defect detection, metric measurements or inventory of equipments, using dedicated software. Therefore, they must be sufficiently bright, sharp and well-resolved to allow visual inspection. Also, every image must be located and oriented as precisely as possible. Since conventional global positioning systems (GPS)-based navigation systems of course do not work in tunnels, other solutions must be sought to accurately locate the recording boat.

The feasibility experiments reported in this paper addressed the objectives of image quality and of location accuracy, with a focus on the second one, which is the most difficult to fulfill. The rest of the paper is organized as follows. In section 2, we give a brief overview of related works. In section 3, we describe the test site, the way a reference model of the tunnel was built and the data recording prototype. In section 4 , we focus on the positioning of the acquisition system. Experimental results are shown and discussed in section 5. Conclusions and future directions are given in section 6 .

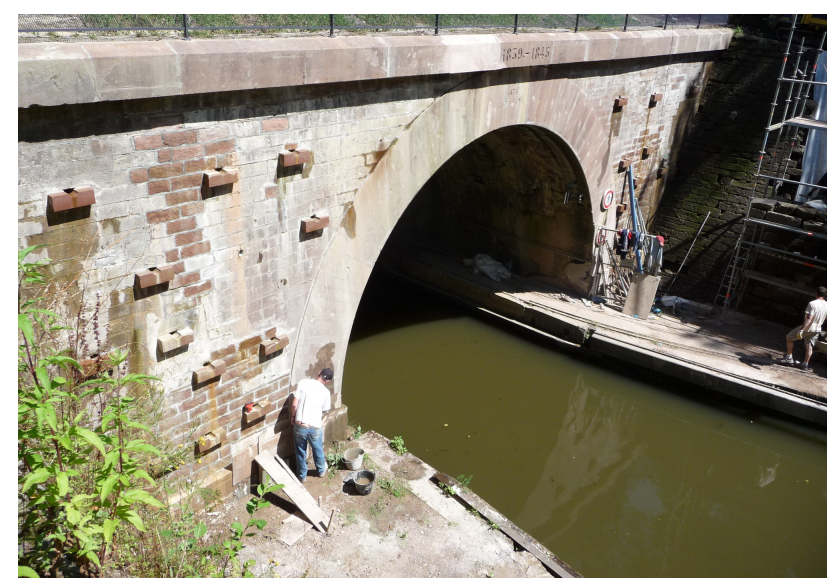

Figure 1: Restoration of Niderviller's tunnel masonry in 2009.

\section{RELATED WORKS}

Dynamic recording for tunnel inspection using 3D (Laser) and/or image data has received an increasing attention during the last few years. Most of the works reported in the literature concern road, subway and railway tunnels. Experimental systems are embedded either on locomotives or carts, or on pick-ups or trucks. To our best knowledge, only one system, combining video cameras and laser pointers onboard a boat, used in Japan for large diameter pipe inspection, has been reported (Kanzaki and Yamaguchi, 2006).

An important issue related to data recording in tunnels is locating the acquisition system in a confined, elongated, and sometimes curved environment. As is well known, Inertial Navigation Systems (INS) suffer from drifts, and must be associated to some external referencing means. Standard techniques for outdoor environments such as GPS-INS data fusion (Grewal $e t$ al., 2007) are inoperative since GPS is unavailable in tunnel. In (Boavida et al., 2012), the INS trajectory was adjusted using control points positioned every $25 \mathrm{~m}$ along a $25 \mathrm{~km}$-long rail- 
way tunnel. In (Müller et al., 2012), total stations implemented every $300 \mathrm{~m}$ inside the Gothard Base Tunnel were used to track reflector prisms mounted on the inspection vehicle. Such conventional trajectography methods require a careful synchronization of all recording devices. They also imply either user interaction or multiple equipments, that both increase the overall cost of inspections.

An alternative to these techniques is to exploit image data. In particular, structure from motion (SFM) is a framework that emerged from the fields of computer vision and digital photogrammetry. It allows reconstructing both the structure of the scene and the camera poses in 3D from an image sequences, which may not be ordered, see e.g. (Agarwal et al., 2011). It relies on image correspondances and on an off-line optimization stage called bundle adjustment, whose computational time and memory requirement increase with the number of images. We refer the reader to (Szeliski, 2010) for a thorough presentation and further references. In contrast, visual odometry (VO) (Scaramuzza and Fraundorfer, 2011, Scaramuzza and Fraundorfer, 2012), which can be seen as a special case of SFM, estimates the 3D motion of the camera in real-time, in a sequential way. In a nutshell, it simplifies the problem by introducing motion estimation into the loop, allowing for dense 3D reconstruction along itineraries in real-time (Geiger et al., 2011). Image-based techniques are promising and seem relevant for our application, since imaging the vaults and sidewalls is the main purpose of the study.

\section{DYNAMIC DATA RECORDING}

\subsection{Experimental site}

Our recording campaigns took place in Niderviller's tunnel, on the Marne-Rhine canal. This canal is a $314 \mathrm{~km}$ long waterway located in the north-east of France. Connected, at its western side, to the lateral canal of the river Marne in Vitry-le-François and, at its eastern side, to the Rhine in Strasbourg, it allows navigating from the Seine basin (Le Havre, Paris) to Germany, Netherlands and eastern Europe. A first investigation, commissioned by king Louis XVI in the early 1780's and conducted by d'Arson and Laffite de Clavé concluded that the project was feasible (Huerne de Pommeuse, 1822). However, the study of the Marne-Rhine canal by engineer B. Brisson really started in 1826 . The construction, leaded by polytechnician C-E. Collignon started in 1838 and the canal was opened to navigation in 1853 . Today, the canal has 166 locks and 4 tunnels. The Vosges massif is crossed via two tunnels located near its lower point, called Col de Saverne, in Niderviller and Arzviller*.

Our experimental site, Niderviller's tunnel (475.45 m long), is shorter than Arzviller's tunnel (2306 m). It is straight, lined with stonework and has a pedestrian path on a ledge. Its construction took place between 1839 and 1845 . Navigation in the tunnel was successively pulled by horses, tugboats and towing locomotives on a narrow railroad, that still exists. Nowadays, boats are selfpropelling and navigate freely in the tunnel. However, navigation is one-way, in alternate mode, and at low speed ( 3 to $6 \mathrm{~km} / \mathrm{h}$, typically). About 7,000 boats, among which a vast majority are pleasure boats, cross the tunnel every year (in 2012). Inspection and restoration campaigns (see fig. 1) are difficult to organize because they require interrupting the traffic.

\footnotetext{
* Note that Arzviller is better known for its inclined plane, located at the eastern side of the tunnel, which, since 1969 , lifts boats on a $44.5 \mathrm{~m}$ high climb, replacing a ladder of 17 locks.
}

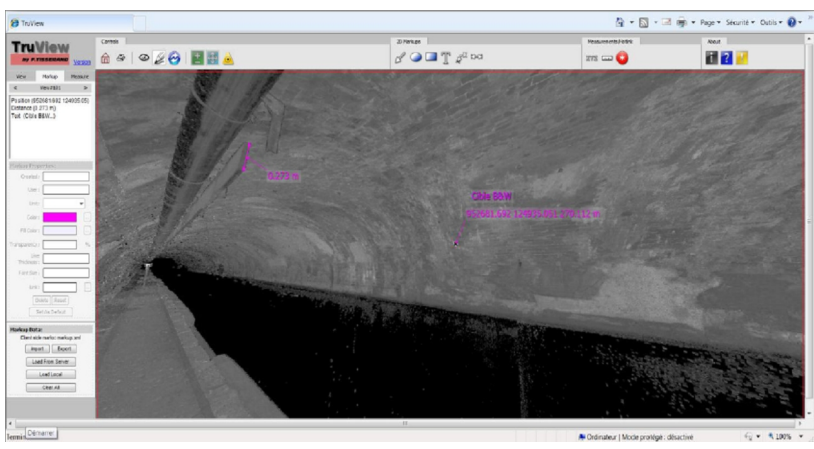

Figure 2: Rendering of the reference model of the tunnel.

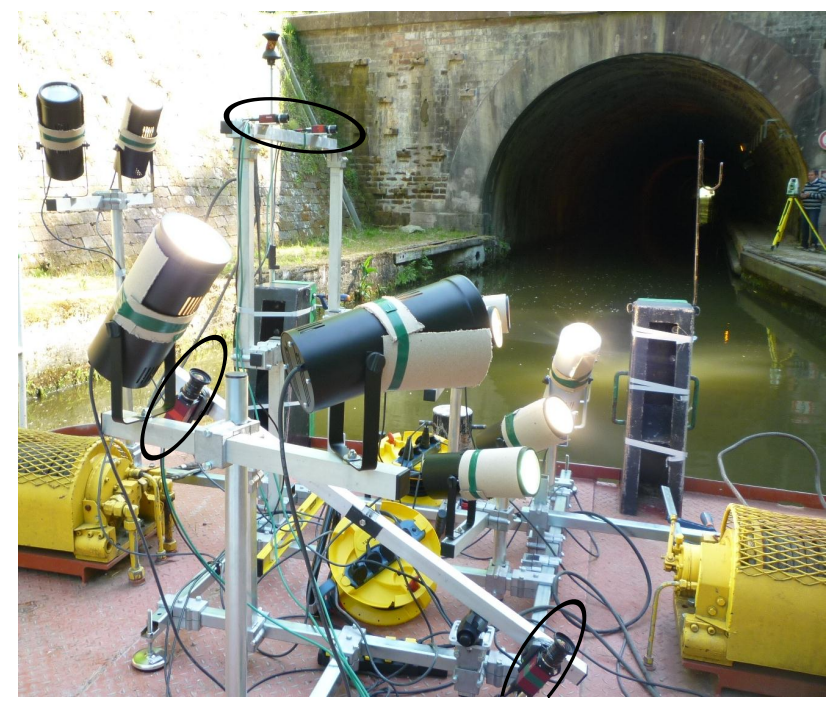

Figure 3: The inspection barge with the modular frame supporting cameras and projectors entering Niderviller's canal tunnel. Black ellipses indicate the positions of cameras.

\subsection{Construction of a reference model of the tunnel}

Prior to our experimentation, a set of carefully georeferenced points was implemented (in RGF 93 and NGF-IGN 69 coordinates) outside and inside the tunnel, using conventional surveying methods. The precision of the planimetric survey was about 1 $\mathrm{cm}$ and the altimetric closure was $3 \mathrm{~mm}$. Then, several additional equipments such as targets, spheres, and visual marks were also placed inside the tunnel. Note that a set of plate, placed every decameter on the sidewall along the bench, was already present, showing the distance from the beginning of the tunnel. Finally, a static 3D surveying of the tunnel was performed using a LEICA C10 laser scanner, which provided a reference point cloud for further precision assessments. More precisely, 15 stations were performed along the ledge of the tunnel (i.e. approximately one every $30 \mathrm{~m}$ ). These high density scans were co-registered using dedicated software, resulting in about 1.5 billion points. The resulting accuracy (difference in position between traditional georeferencing and the 3D model) over 100 control points is less than $1.7 \mathrm{~cm}$. The reader is referred to (Charbonnier et al., 2013), in the same archive, for further details. A rendering of the model using LEICA's TruView software is shown on fig. 2.

\subsection{Recording prototype}

The prototype that has been used for our recording campaigns is based on a modular structure that can be equipped with different kinds of sensors and lightings. It is basically a frame of square sectional aluminium tubes assembled with adjustable chucks. Silent-blocks are used to insulate the structure from vibrations of 

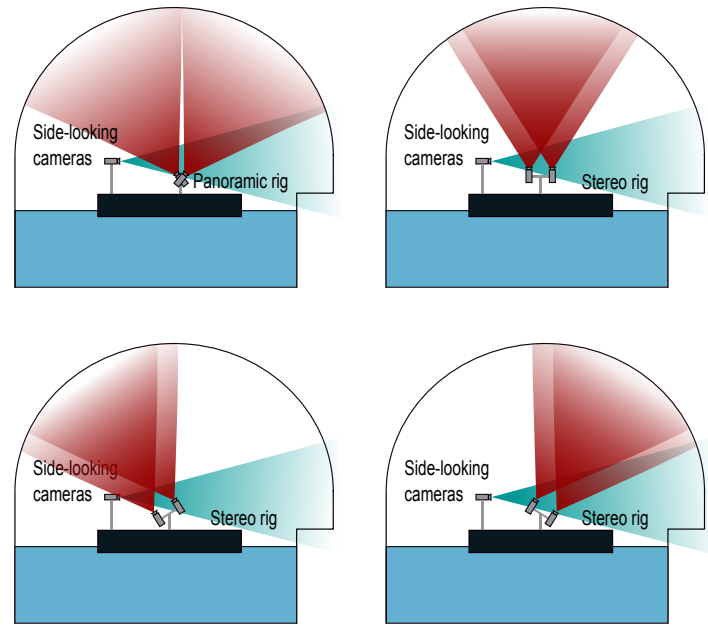

Figure 4: Schematic view of the tunnel and the barge featuring the camera configurations. Top left: panoramic, top right: stereo $\left(0^{\circ}\right)$, bottom left: stereo $\left(32^{\circ}\right)$ and bottom right: stereo $\left(-32^{\circ}\right)$.

the barge. Vertical tubes are fixed on the frame, to support projectors and cameras, as can be seen on fig. 3 . Note that a careful static geometric survey of the frame was performed, to determine the transformations between the barge and the different elements of the prototype, to make location tasks easier.

Several kinds of projectors were first tested in laboratory. Metalhalide lamps with electronic ballast were chosen for their good performance, relatively low electrical consumption and low price. In the tunnel, 10 projectors are used. Their orientations are first arranged in laboratory, but thanks to the modularity of our structure, they can be adjusted on-site to make illumination as uniform as possible.

To record images, we take commercial high-resolution industrial cameras, namely Pike F210C $(1920 \times 1080$ pixels $)$ and Marlin F145C (1392 × 1040 pixels), from Allied Vision Tech (AVT). More specifically, we use a pair of each model, in the following way: the Marlin cameras are placed on the left side of the boat, facing the right side wall. They are fixed on a bar, as parallel to each other as possible, to form a stereo rig. The advantage of considering a stereo device is that 3D measurements are possible, once the system is calibrated (Szeliski, 2010). The Pike cameras can be arranged either to form a stereo rig or to form a panoramic rig directed towards the vault. The axis of the stereo rig can be oriented along 3 angles with respect to the vertical $\left(-32^{\circ}, 0^{\circ}\right.$ and $32^{\circ}$ ) and the aperture angle is about $75^{\circ}$, so 3 passes are necessary to acquire images of the whole vault. In panoramic configuration, the aperture angle is nearly $145^{\circ}$, so a single pass is sufficient, but 3D measurements are not possible. The camera configurations are summarized on fig. 4 .

Synchronism is a keypoint for stereovision, whose principle relies on finding corresponding points in both views of the same scene. It is also interesting to synchronize both pairs of cameras to obtain complementary views (of the vault and side wall). To this aim, we use an external trigger device, that generate pulses at regular time intervals. Note that, contrary to railway or road tunnels, it is not possible here to trigger the acquisitions by distance, since no reliable odometric sensor is directly available.

Finally, since the images are taken from a moving barge, they may suffer from motion blur. In order to avoid this effect, one has to adapt the exposure time and the aperture of the diaphragm.

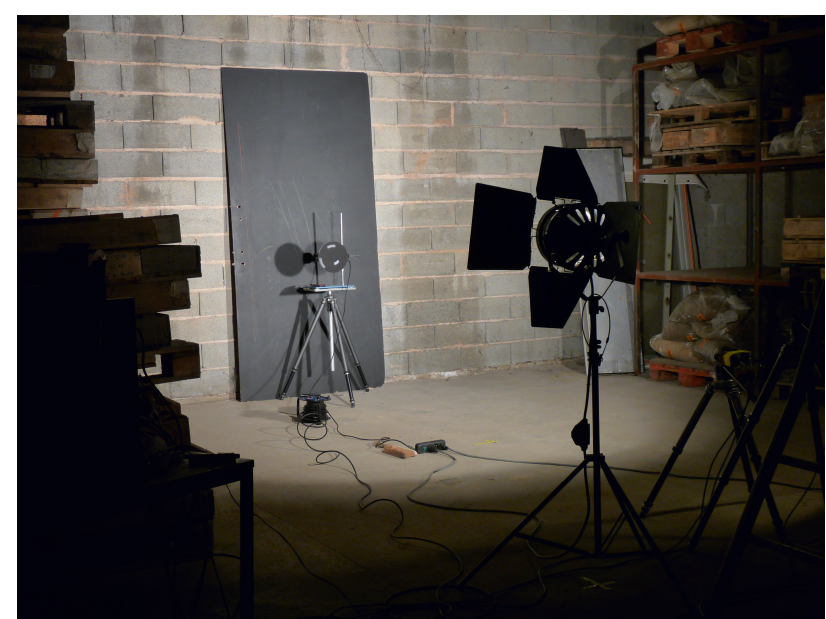

Figure 5: Setting up electronic and optical parameters in lab, using a rotating disk under artificial illumination.

Of course, the fact that images are taken under artificial lighting enforces some constraints on this adjustment. Therefore, we performed a laboratory experiment (see fig. 5) which consisted in taking images of a rotating disk (with a speed close to $1 \mathrm{~m} . \mathrm{s}^{-1}$ ) in the same illumination conditions as in the tunnel. The optical and electronic parameters were then chosen to optimize the image quality.

\section{LOCATION METHODS}

Several positioning methods, with different capabilities, were tested by exploiting the recorded data off-line: simplified visual odometry (using single camera or stereo rigs) to position the barge in curvilinear abscissa; photogrammetry, which provides both a $3 \mathrm{D}$ model of the structure and camera locations and orientations; inertial navigation and tacheometric tracking.

\subsection{Trajectography}

During our experiments, we tested two ways of measuring the trajectory of the barge. The first one relies on external means. More specifically, tacheometers were placed at the ends of the tunnel for tracking the barge, which was equipped with targets. For the first campaign, 3 reflector prisms were used, in order to obtain both position and orientation information. For the second one, a single prism was considered. Synchronization with the other devices was made using a reference timer for the first experiment, and using the speaking clock, for the second one.

We also tested an on-board solution: a commercial navigation system, which combines a differential GPS (Ashtech Proflex), an inertial navigation system (IXSEA Phins), and a Doppler log (RDI Explorer PA). GPS data is used to initialize a Kalman filter that integrates inertial and Doppler data.

\subsection{Simplified visual odometry (SVO)}

As explained in section 2, there are several ways of computing the successive positions of the imaging system from an image sequence. Under some simplifying assumptions about the motion of the boat, its curvilinear abscissa can be obtained from images of the side walls in a more or less automatic way.

Interactive SVO The simplest procedure implies user interaction and relies on the assumption that the speed of the barge is constant by intervals. It requires that the distance between successive decametric plates, which is never exactly $10 \mathrm{~m}$, has been 


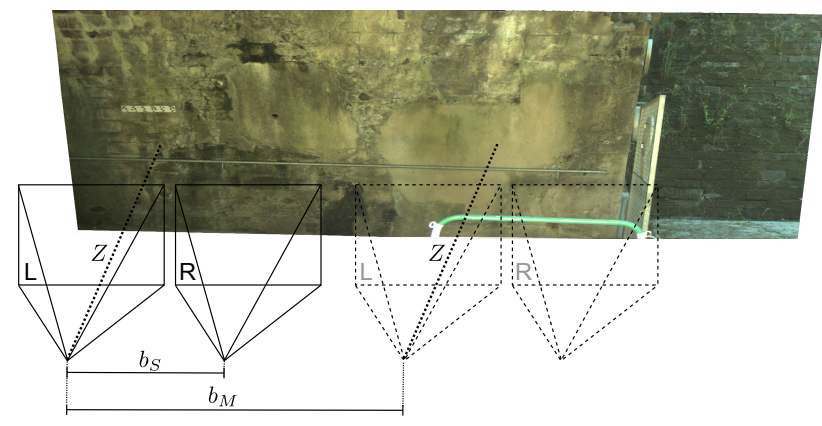

Figure 6: Automatic simplified visual odometry configuration. Solid lines: stereo rig at time $t$. Dashed lines: stereo rig at times $t-1$. The cameras are supposed to be parallel to the side wall.

measured beforehand. More precisely, the user points out all images were a decametric plate is the closest to a pre-defined landmark (typically the center of the image). Then, the interval between successive images is obtained as the ratio between the inter-plate distance and the number of images. This procedure is valid for single-camera image sequences, but stereovision can be used to correct the measure from the shift between the center of the plate and the center of the image.

Automatic SVO Stereovision can be exploited in a more automatic way. Once the stereo rig is calibrated, it is possible to rectify images, i.e. to transform them as if they were taken by similar, perfectly aligned cameras, see (Szeliski, 2010) for details, and to correct them from geometric distorsion. In this configuration, it is well known that depth, $Z$, is an inverse function of the disparity $d_{S}$, which is the apparent displacement between images of the scene:

$$
Z=\beta \frac{b_{S}}{d_{S}}
$$

where $\beta$ is a scale ratio and $b_{S}$ is the stereo base, i.e. the distance between cameras. Both parameters are estimated by calibration. Let us consider a pair of successive images taken by one camera of the stereo rig and assume that the motion of the boat is parallel to the base line of the imaging system. This defines a new stereo configuration, with unknown base distance, $b_{M}$. Then, we have:

$$
Z=\beta \frac{b_{M}}{d_{M}},
$$

where the image motion $d_{M}$ can be seen as a disparity and the base distance $b_{M}$ is the motion of the system. Assuming that $Z$ remains constant while the barge moves, we obtain:

$$
b_{M}=\frac{d_{M}}{d_{S}} b_{S},
$$

which means that the motion of the system (in meters) can be deduced from the displacement (in pixels) between left and right images of a stereo pair, and the displacement (also in pixels) between successive left (or right) images. In practice, $d_{M}$ and $d_{S}$ are computed using phase correlation (Szeliski, 2010), which is fast and easy to implement.

\subsection{Photogrammetric modeling}

The most classical method in structure-from-motion is bundle adjustment. To apply this technique, we consider the images of the right camera of the stereo rigs and we use a commercial software, namely PhotoModeler Scanner (PMS). The program first searches interest points in the images. Then it looks for correspondences between these points (homologous points). Finally, it estimates both the 3D structure of the scene, up to a scale factor, and the successive positions and orientations of the cameras.

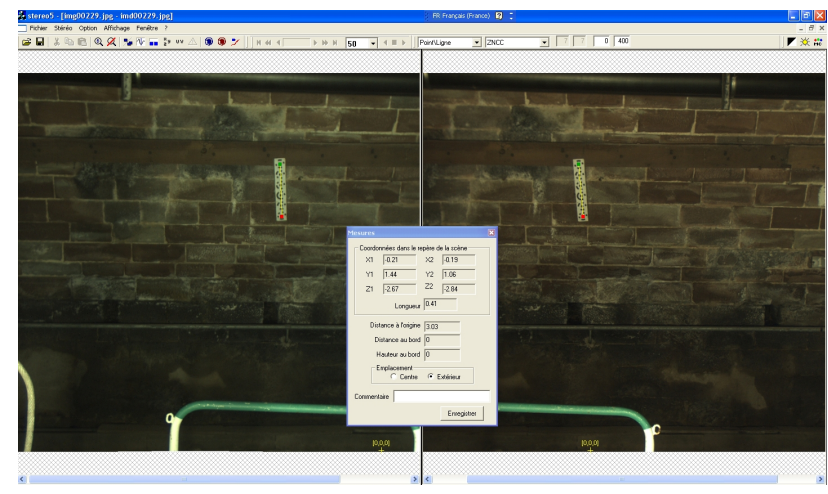

Figure 7: Example of measurement on a side wall using a stereo pair. The real distance between points on the bar-coded target is $42 \mathrm{~cm}$. The measured distance is $41 \mathrm{~cm}$.

Geo-referenced points can be used to remove scale ambiguity and to fix the geometry of the model. The reader is referred to (Charbonnier et al., 2013) in the same archive for more details about the construction of the 3D model.

\section{RESULTS AND DISCUSSION}

\subsection{Images}

The feasibility study confirmed that recording images of the side walls and of the vault is not a real difficulty. Of course, special care must be taken to synchronization and to the quality of illumination. In our experiments, the barge was moving at about $1 \mathrm{~m} . \mathrm{s}^{-1}$. We acquired images at a frame rate of $1 \mathrm{~Hz}$ and $5 \mathrm{~Hz}$. This results in a distance interval of approximately $1 \mathrm{~m}$ (resp. $20 \mathrm{~cm}$ ) between successive acquisitions, which corresponds to an overlap of $75 \%$ (resp. $95 \%$ ) between successive images. In our configuration, 3 passes were necessary to acquire images of the whole vault, but a single pass would be made possible by multiplying the number of cameras.

Stereo images can be exploited using dedicated software to provide 3D measurements (see e.g. fig. 7). Moreover, 3D models can be constructed from single-camera based image sequences, using PMS.

The pairs of images taken by the panoramic rig are combined to create panoramas of the vaults, which is known as image stitching (Szeliski, 2010). Both images are projected onto a cylinder, which seems well adapted to the context. Since images of the panoramic rig differ by a rotation, their cylindric projections are linked by a translation. This parameter is robustly estimated from corresponding points. The displaced images are then blended to form the panorama. In practice, we use the Hugin software to estimate the parameters of the transformation for a pair of representative images. Then, we apply the same transform to each pair of the sequence using the PanoTools library. This process is illustrated on fig. 8. As can be seen on the figure, the illumination of the vault is not as uniform as expected. Moreover, some parts of the prototype create shadows. To correct these effects, we make a "flat field" image by taking the overall mean of panoramas. The resulting gray level image is blurred in order to smooth the stripes resulting from the presence of masonry. Finally, we apply the flat field image to each panorama, which results in corrected images. This procedure can be applied whatever the inter-distance between successive image pair acquisition. However, we found that browsing through the panorama sequence is more fluid when its is about $20 \mathrm{~cm}$. 

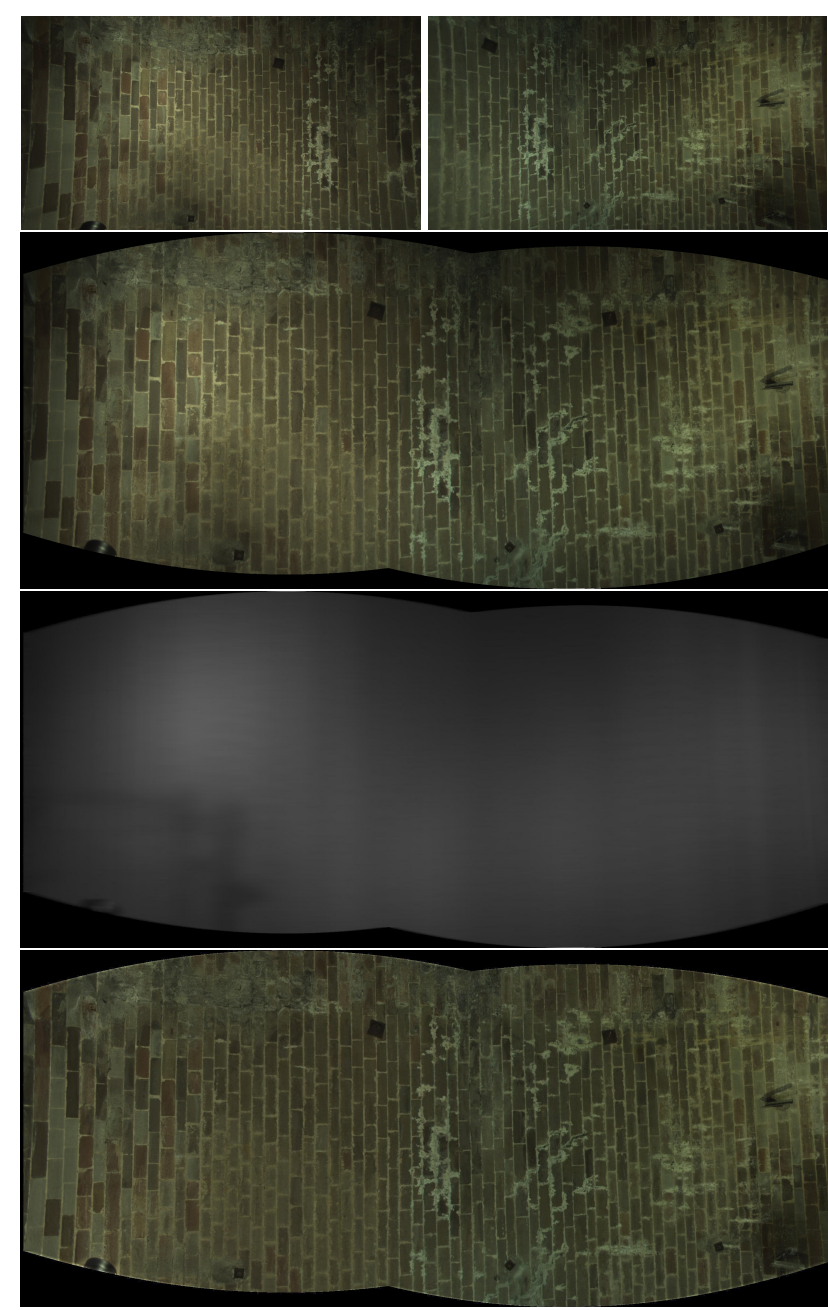

Figure 8: Example of panoramic image. From top to bottom: original images, combined image, "flat field" image exhibiting shadows and illumination variations, corrected panorama.

\subsection{Location of the vision system}

In this section, we analyze the pros and cons of each location method in light of our experiments. Comparisons mostly rely on the result of the second experiment, in which all 4 solutions were simultaneously tested. During this run, the boat followed a straight trajectory, excepted by the end of the tunnel, where it slowed down and was drifted towards the ledge by its own wave. This resulted in a loop in the planimetric trajectory and, also in altimetric perturbations.

Tacheometric tracking During the first experiment, 3 prisms were mounted on the boat. We observed confusions that were probably due to the limited observation angles. In the second experiment, only one prism was used with 4 tacheometers. As can be seen on Fig. 9, the tracking is not perfect, which results in peaks in the inter-image distance graph. Such peaks might be smoothed out easily from the curve. Another problem we faced was synchronization with other devices: the internal clock of tacheometers is apparently not very accurate nor stable. Finally, we found that the useful range of tacheometers is limited to about $300 \mathrm{~m}$, which is consistent with observations of (Müller $e t$ $a l ., 2012)$. This means that using this solution in longer or curved tunnels would require multiplying the number of tacheometers, hence raising the difficulty of synchronization. These drawbacks are counterbalanced by a good altimetric precision: the altitude variations were less than $15 \mathrm{~cm}$, with a maximum near the end of the tunnel, where the trajectory of the boat was perturbed.

Inertial navigation The tested system is based on a Kalman filter initialized by a GPS, whose signal was highly perturbed because the canal is in a trench before the tunnel (see fig. 3). Moreover, inside the tunnel, the inertial system strongly drifted $(1 \mathrm{~m}$ in planimetry and $2 \mathrm{~m}$ in altimetry). However, it was possible to adjust the trajectory thanks to the loop near the end of the tunnel. After correction, the planimetric deviation with respect to PMS results (see below) was less than $8 \mathrm{~cm}$ and the altitude variations, less than $15 \mathrm{~cm}$. This experiment shows that, although the system is appealing since it is autonomous, it requires a good initialization and periodic corrections of the drift using either external measurements, as in (Müller et al., 2012) or manually pointed control points, as in (Boavida et al., 2012).

Photogrammetry with PMS Image-based method are interesting because they do not involve external devices. Moreover, structure from motion (implemented by PMS) provides both the trajectory of the imaging system and a 3D reconstruction of the tunnel. However, these methods are computationally demanding. For example, to compute a 3D model of the tunnel with a high performance PC, 9 sub-models had to be computed and assembled. Moreover, there are many possibilities in terms of parameter setup, and some experience is needed. Finally, finding correspondances between views requires that images are sufficiently textured. Note that, due to error accumulation, the resulting model was curved. To tackle this problem, several reference points had to be included in the computations. The accuracy of the resulting 3D model was first assessed by measuring the distances between couples of points separated by $8 \mathrm{~m}$ each. The error was between -0.9 and $1.9 \mathrm{~cm}$. Then, a global comparison of point clouds between the reference model and the model estimated with PMS shown that differences were less than $5 \mathrm{~cm}$ (see (Charbonnier et al., 2013), this proceedings, for more details). These rather good figures, however, concern the 3D model, not the trajectory. Assessing the precision of the trajectory itself is impossible since we have no reference. The method we use is consistent with the application: since the aim of visual inspection is to locate defects or equipments, we compare the coordinates of several points, pointed out by an operator in the reference (Laser) point cloud and in the PMS model. Over 9 such control points, the average difference is $2 \mathrm{~cm}$, with a maximum of $11 \mathrm{~cm}$.

Simplified VO The first two simplified odometry methods we evaluated are interactive, see Sec. 4.2. To assess the accuracy of these techniques, we use the same method as for photogrammetry: 15 control points were pointed in the $3 \mathrm{D}$ model computed by PMS and 9 were selected in the reference lasergrammetric model. Their positions were also computed in single or stereo image sequences. The results are given in Tab. 1. Not surprisingly, the single-image based method is rather inaccurate. Introducing $3 \mathrm{D}$ corrections thanks to stereovision significatively reduces the mean and maximum differences.

\begin{tabular}{|c|c|c|c|c|}
\hline \multirow{2}{*}{$\begin{array}{c}\text { SVO } \\
\text { method }\end{array}$} & \multicolumn{2}{|c|}{ vs. PMS } & \multicolumn{2}{c|}{ vs. Laser } \\
\cline { 2 - 5 } & mean $(\mathrm{m})$ & $\max (\mathrm{m})$ & mean $(\mathrm{m})$ & $\max (\mathrm{m})$ \\
\hline Single & 0.68 & 1.08 & 0.79 & 1.18 \\
Stereo & 0.17 & 0.34 & 0.13 & 0.36 \\
Automatic & 0.19 & 0.50 & 0.14 & 0.31 \\
\hline
\end{tabular}

Table 1: Absolute differences between control points extracted from images, and pointed out in a reference model (Laser model or PMS model).

The third method, which is fully automatic, reaches almost the same performance as the stereo-based manual technique. We also compared the $Z$ coordinate with a manual measure made 


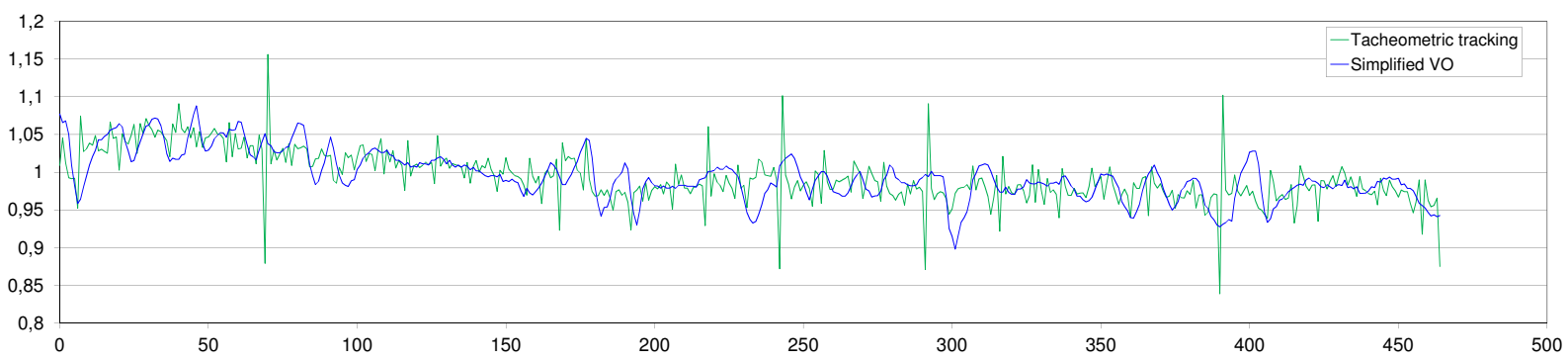

Figure 9: Inter-image distance (m) vs. curvilinear abscissa (m): comparison between tacheometric tracking and automatic simplified visual odometry.

from the PMS model, for 48 positions along the tunnel. The mean difference is $4.4 \mathrm{~cm}$ (with a maximum of $14.4 \mathrm{~cm}$ ). For comparison, the mean is $6.2 \mathrm{~cm}$ for manual stereovision, with a maximum of $13.3 \mathrm{~cm}$ ). We found that phase correlation works better with $95 \%$ overlap than with $75 \%$ overlap. The value of the correlation coefficient gives an indication about the quality of the image correspondance. Correlation failures result in outliers in the inter-image distance graph, which can be filtered out easily, as was done for the plot shown on Fig. 9, where inter-image distances from automatic visual odometry are compared to interimage distances obtained by projecting the speed vectors from tacheometric tracking onto the axis of the tunnel. As can be seen, the curves are rather similar. Incorporating a dynamic model of the boat in the estimation should provide smoother results. Finally, the drift of the method was evaluated to $4.5 \%$ o (i.e. 2.14 $\mathrm{m}$ over the tunnel). Using registration points in a post-processing step will tackle this problem. These promising results can be seen as a first step towards considering more complete motion models than translation.

\section{CONCLUSIONS AND FUTURE WORK}

In this paper, we have introduced a modular prototype dedicated to dynamic data acquisition in canal tunnels and reported the results of preliminary tests that were performed in Niderviller's tunnel. Not surprisingly, they shown that synchronism is an important issue in a multiple-sensor context. Also, it appears that a minimal equipment of the tunnel is required since, whatever the method, landmarks or control points are required. Designing an image acquisition under artificial illumination system is not a difficult issue. The experiments shown that it is better to consider relatively small distance interval between images. Locating the imaging system along the tunnel is a much more difficult problem. Tacheometric tracking is limited by the range of these apparatus and the difficulty to synchronise them with other acquisition devices. Inertial navigation systems exhibit drifts, that may be corrected at the price of an accurate registration, which cannot be provided by GPS, but could be obtained from tacheometric measurements or control points. Finally, an interesting strategy to avoid combining different systems seems to exploit image data as much as possible. Photogrammetric techniques, such as bundle adjustment provide both a 3D model of the infrastructure as well as the position and orientation of cameras. However, they are very demanding in terms of user interaction, computational time and memory requirements. Visual odometry, which exploits motion contraints, is increasingly used in mobile-mapping and seems a promising way to make the procedure more accessible. The results obtained here, with a simplified implementation, seem sufficient to reach a decimetric precision. Adapting more sophisticated methods to the particular geometry of canal tunnels, in order to increase the precision of the system will be addressed in a near future.

\section{ACKNOWLEDGEMENTS}

The authors would like to thank Voies Navigables de France and the Centre d'Etudes des Tunnels for funding this project. Many thanks, also, to the students of INSA de Strasbourg who contributed to the project, and to P.-Y. Scordia and B. Singer for providing historical and statistical information.

\section{REFERENCES}

Agarwal, S., Furukawa, Y., Snavely, N., Simon, I., Curless, B., Seitz, S. and Szeliski, R., 2011. Building Rome in a day. Comm. ACM 54(10), pp. 105-112.

Boavida, J., Oliveira, A. and Santos, B., 2012. Precise long tunnel survey using the Riegl VMX-250 mobile laser scanning system. In: Riegl LiDAR 2012, Riegl, Orlando, Florida, USA.

Charbonnier, P., Chavant, P., Foucher, P., Muzet, V., Prybyla, D., Perrin, T., Grussenmeyer, P. and Guillemin, S., 2013. Accuracy assessment of a canal-tunnel 3D model by comparing photogrammetry and laserscanning recording techniques. In: XXIV International CIPA Symposium, Strasbourg.

Geiger, A., Ziegler, J. and Stiller, C., 2011. Stereoscan: Dense 3D reconstruction in real-time. In: IEEE Intelligent Vehicles Symposium (IV), pp. 963-968.

Grewal, M., Weill, L. and Andrews, A., 2007. Global Positioning Systems, Inertial Navigation, and Integration. 2nd edn, John Wiley \& Sons, Hoboken, NJ, USA.

Huerne de Pommeuse, M., 1822. Des canaux navigables considérés d'une manière générale. Bachelier et Huzard, Paris.

Kanzaki, T. and Yamaguchi, S., 2006. Current situation and issues concerning the maintenance and management of underground lifeline structures in Japan. - Evolving toward the introduction of information technology, automation and robotization. In: The 23rd International Symposium on Automation and Robotics in Construction 2006, Japan Society of Civil Engineers, Tokyo, Japan, pp. 293-298.

Müller, U., Kuhn, P. and Kadner, G., 2012. Potential savings in motion. World tunelling, (March 2012), pp. 25-27.

Scaramuzza, D. and Fraundorfer, F., 2011. Visual odometry - part I: The first 30 years and fundamentals. Robotics \& Automation Magazine, IEEE 18(4), pp. 80-92.

Scaramuzza, D. and Fraundorfer, F., 2012. Visual odometry - part II: Matching, robustness, optimization, and applications. Robotics \& Automation Magazine, IEEE 19(2), pp. 78-90.

Szeliski, R., 2010. Computer Vision: Algorithms and Applications. 1st edn, Springer-Verlag New York, Inc., New York, NY, USA. Available online at http: \\szeliski.org \Book. 